\title{
DT-CWT Robust Filtering Algorithm for the Extraction of Reference and Waviness from 3-D Nano Scalar Surfaces
}

\author{
Zhi Ying. Ren ${ }^{1,2}$, ChengHui. Gao ${ }^{1,2}$, GuoQiang. Han ${ }^{1}$, Shen Ding ${ }^{1}$, JianXing. Lin ${ }^{1}$ \\ ${ }^{1}$ School of Mechanical Engineering and Automation, Fuzhou University, Fuzhou 350108, China \\ ${ }^{2}$ The tribology Institute of Fuzhou University, Fuzhou 350108, China, gch@fzu.edu.cn
}

Dual tree complex wavelet transform (DT-CWT) exhibits superiority of shift invariance, directional selectivity, perfect reconstruction (PR), and limited redundancy and can effectively separate various surface components. However, in nano scale the morphology contains pits and convexities and is more complex to characterize. This paper presents an improved approach which can simultaneously separate reference and waviness and allows an image to remain robust against abnormal signals. We included a bilateral filtering (BF) stage in DT-CWT to solve imaging problems. In order to verify the feasibility of the new method and to test its performance we used a computer simulation based on three generations of Wavelet and Improved DT-CWT and we conducted two case studies. Our results show that the improved DT-CWT not only enhances the robustness filtering under the conditions of abnormal interference, but also possesses accuracy and reliability of the reference and waviness from the 3-D nano scalar surfaces.

Keywords: 3-D nano scale, DT-CWT robust filtering, reference, waviness.

\section{INTRODUCTION}

$\mathrm{E}_{\mathrm{p}}$ NGINEERING SURFACES may be described by the parameters of reference, waviness and roughness. Filtration has always been the basis of the assessment of 3-D surface parameters, by which the surface features are extracted from the roughness surface and the accuracy of the surface features is also indirectly affected by it. With the development of planar technology and miniaturization, conventional filters like the Gaussian filter and B-spline filter [1] often do not meet the required standard when a multi-scale topography is present in an ultra-precision surface [2].

Wavelet transform employs space-frequency windows to divide an original surface into different scale-frequency components and this transform has been widely used in the field of 3-D surface morphology characterization. But Wavelet transform has many deficiencies, such as phase distortions, shift-variant problems and outlier distortions [3-6]. In particular, the appropriate wavelet basis and the steps of wavelet decomposition have not been studied in 3-D surface characterization and this lack of information directly restricts the application of wavelet technology. To solve these problems, Kingsbury [7] proposed DT-CWT based on a complex wavelet model. This model minimizes the distortion caused by phase and shift-variants. DT-CWT has many other advantages, such as directional selectivity, perfect reconstruction and limited redundancy. However, in practice, there are many abnormal signals in the measured surface, the filtered surface is pulled down and the residual surface is deformed in the opposite direction, which affects the accuracy of the reference surface [2]. To solve this problem, we demonstrate an improvement to DT-CWT by implementation of a bilateral filter. A simulation test shows that our improved filtering method could enhance the restriction of outliers in DT-CWT.

\section{MATERIALS USED IN THE ANALYSIS}

\section{A. Dual tree complex wavelet (DT-CWT)}

Fig.1. illustrates the standard DT-CWT decomposition and reference reconstruction.
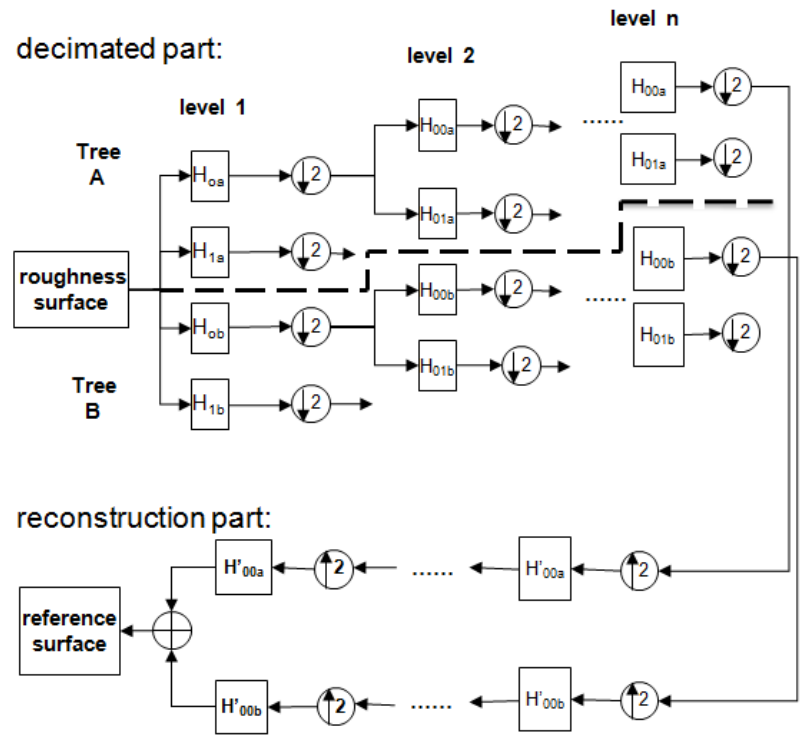

Fig.1. The frame of DT-CWT decomposition and the reference reconstruction.

From the above figure, the DT-CWT comprises two trees, A and $\mathrm{B}$, by which we can obtain the real and imaginary parts of low-frequency coefficients. Where the $H_{0 a}, H_{1 a}$ and $H_{0 b}, H_{1 b}$, comprised by Q-shift filters are the decimated filter; $H_{0 a}^{\prime}$ and $H_{1 b}^{\prime}$ are the reconstruction filters; $\uparrow$ is the upper sampling; $\downarrow$ is the down sampling. Since the Q-shift filter is not in linear phase, it must use different sampling rates in both trees to achieve linearity. Therefore, tree $\mathrm{A}$ is designed to include a group delay of $1 / 4$ sample and tree B guarantees $3 / 4$ samples by using the time reverse of the tree A filter. In this way, the sampling site in tree B is just between the sites in 
tree A. The reconstruction filter of tree $\mathrm{A}$ is the reverse of the corresponding filter in tree $\mathrm{B}$. The above contributes to the shift invariance.

Compared with the conventional discrete wavelet, the DT-CWT may avoid shift-variant problems and phrase distortions, but it fails to avoid outlier distortions. In complex wavelet transform, the two-dimensional signal can be expressed as a combination of 2-D wavelet function and sub-band function of six directions [8]:

$$
f(s)=\sum_{k \in Z^{2}} a_{J, L} \phi_{J, L}(s)+\sum_{b \in B} \sum_{j \leq J} \sum_{l \in Z^{2}} d_{J, L}^{b} \psi_{J, L}^{b}(s)
$$

Where $S\left(s_{1}, s_{2}\right) ; \mathrm{B}=\left\{ \pm 15^{\circ}, \pm 45^{\circ}, \pm 75^{\circ}\right\}$; each sub-band function can be expressed as follows:

$$
\begin{gathered}
\psi^{+15^{\circ}}(s)=\phi\left(s_{1}\right) \psi\left(s_{2}\right), \psi^{+45^{\circ}}(s)=\psi\left(s_{1}\right) \psi\left(s_{2}\right), \\
\psi^{+75^{\circ}}(s)=\psi\left(s_{1}\right) \phi\left(s_{2}\right), \psi^{-15^{\circ}}(s)=\phi\left(s_{1}\right) \bar{\psi}\left(s_{2}\right), \\
\psi^{-45^{\circ}}(s)=\psi\left(s_{1}\right) \bar{\psi}\left(s_{2}\right), \psi^{-75^{\circ}}(s)=\psi\left(s_{1}\right) \bar{\phi}\left(s_{2}\right),
\end{gathered}
$$

Due to the six directions of sub-bands, DT-CWT can characterize the surface topography accurately. Although DT-CWT improves output accuracy, computational redundancy is also increased.

In the ideal situation, random roughness will comply with a Gaussian ordinate distribution. But in practice, because of the freak interference in the measured surface, the reference surface (or waviness surface) will be pulled down or up. In sum, the outlier distortions restrict the application of DT-CWT in practical engineering.

\section{B. Bilateral filtering}

As a spatial filter, the bilateral filter is also an iterative and nonlinear filter [9]. When there are no abnormal features, the reference from the bilateral filter is the same as that from a linear filter. On the contrary, the influence of abnormal features is suppressed. The one-dimensional reference $h(x)$ can be expressed as follows [10]:

$$
\begin{array}{r}
h(x)=k^{-1}(x) \int_{-\infty}^{\infty} f(\varepsilon) c(\varepsilon, x) s(f(\varepsilon), f(x)) d \varepsilon \\
k(x)=\int_{-\infty}^{\infty} c(\varepsilon, x) s(f(\varepsilon), f(x)) d \varepsilon
\end{array}
$$

Where $c(\varepsilon, x)$ is the geometric weight between the neighborhood center $x$ and a nearby point $\varepsilon$ (Gaussian type: $\left.c(\varepsilon, x)=\exp \left(-\|\varepsilon-x\|^{2} / 2 \delta_{d}{ }^{2}\right)\right) ; s(f(\varepsilon), f(x))$ is the photometric similarity function between the point $x$ and a nearby point $\varepsilon$, Gaussian type:

$c(\varepsilon, x)=\exp \left(-\|f(\varepsilon)-f(x)\|^{2} / 2 \delta_{r}{ }^{2}\right), k(x)$ is a normalization factor; $f(\varepsilon)$ is the measures profiles; and $h(x)$ is the reference line.

From (3) and (4), we know that the difference between bilateral filtering and Gaussian filtering is the photometric similarity function $s(f(\varepsilon), f(x))$. We may use this function to compensate for the geometric approach weights $c(\varepsilon, x)$. In order to illustrate this more clearly, we simulated a 3-D surface with an abnormal point as shown in Fig.2., and with the weight distribution of the central point shown in Fig.3.

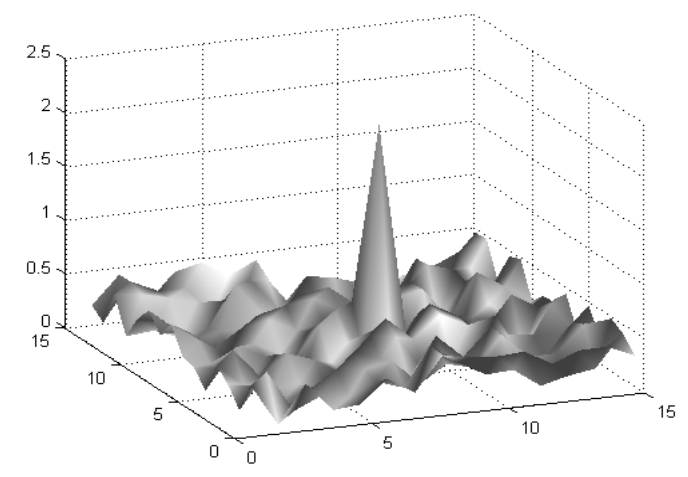

Fig.2. 3-D rough surface containing abnormal point.

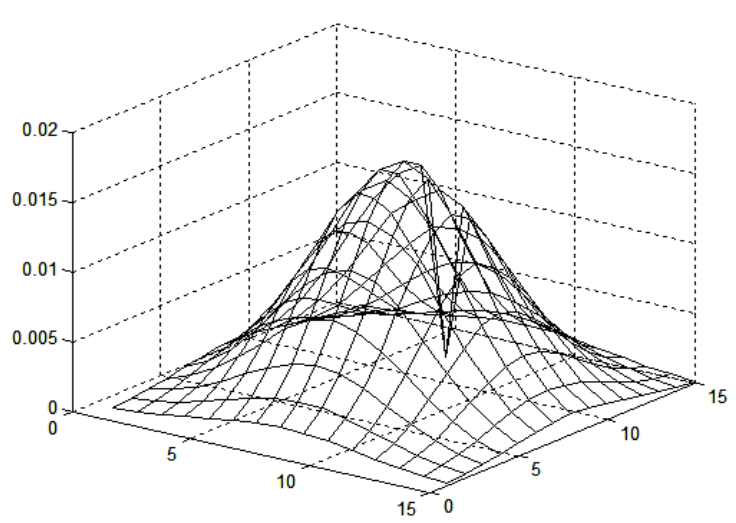

Fig.3. Weight window of the bilateral filter.

In the time-space domain, the site of 3-D surface signal $f(x, y)$ is similar to a function field, in that the height of each sample point is like the value of the function. In order to improve precision of the surface characterization, bilateral filtering introduces the photometric similarity function into the filter kernel, to reasonably allot the weight of each point in the filter window. When the magnitude difference of the sample points and the center of filter is large, it reduces the corresponding weight distribution of the sample points. This allows for the avoidance of reference distortions containing abnormal points in each window. Due to its features, bilateral filtering has an irreplaceable practical advantage. However, in computational efficiency (as is also true for spatial and self-adaptive filtering), bilateral filtering is less useful than the dual tree complex wavelet, because the latter can use FFT to improve its computing speed. Therefore, the dual tree complex wavelet can not only maintain converging velocity and computing efficiency, but may also ensure the accuracy of the roughness parameters that first warranted its introduction into the tree complex wavelet. 


\section{The improved algorithm}

In this paper, the new bilateral filter algorithm was mainly used in 3-D surface reference characterization. For a given 3-D surface, the algorithm defines the measured surface as composed of reference, waviness, and roughness parameters. The measured surface $f(x, y)$ can be expressed as follows:

$$
f(x, y)=s(x, y)+\varepsilon(x, y)
$$

Where $s(x, y)$ is the objective surface (mainly reference or waviness); and $\varepsilon(x, y)$ is the roughness surface. The aim of this article is to smooth the surface roughness, use the low frequency parts of the surface to reconstruct the reference surface $s(x, y)$, and filter noisy signal $\varepsilon(x, y)$ in the noise surface $f(x, y)$ as much as possible to restore the ideal datum $s(x, y)$. Fig.4. illustrates the frame of the algorithm described in this paper.

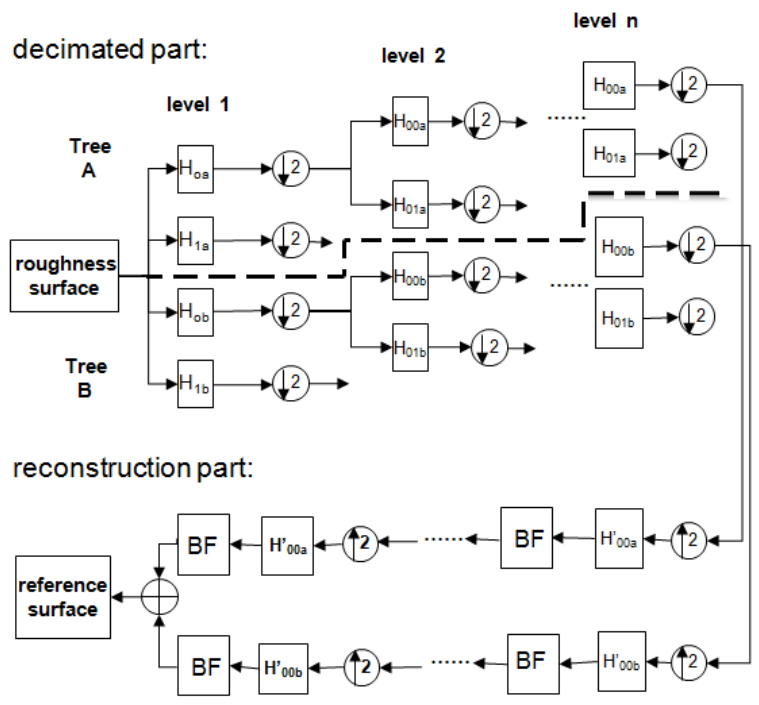

Fig.4. Frame of improved tree complex wavelet filtering.

Where $H_{0 a}, H_{1 a}, H_{0 b}, H_{1 b}, H_{0 a}^{\prime}$ and $H_{0 b}^{\prime}$ are defined as above. BF is the bilateral filter. The process of the decimated part is the same as that in DT-CWT. The major difference is that there is a process of bilateral filtering that occurs between the reconstruction steps. In order to ensure the shift-invariance and the authenticity of the filtered reference, a bilateral filter allows the Gaussian function to be used for practical applications.

To characterize the 3-D surface, the bilateral filtering and its parameters can be expressed as follows:

$$
\begin{gathered}
h(x, y)=k^{-1}(x, y) \int_{-\infty}^{\infty} \int_{-\infty}^{\infty} f(\zeta, \eta) c(x-\zeta, y-\eta) \\
\cdot s(f(x, y), f(x-\zeta, y-\eta)) d \zeta d \eta \\
k(x, y)=\int_{-\infty}^{\infty} \int_{-\infty}^{\infty} c(x-\zeta, y-\eta) s(f(x, y), \\
f(x-\zeta, y-\eta)) d \zeta d \eta
\end{gathered}
$$

Close function $c(x-\zeta, y-\eta)$ and photometry similarity function $s(f(x, y), f(x-\zeta, y-\eta))$, respectively, are expressed as follows:

$$
\begin{aligned}
& c(x-\zeta, y-\eta)=k \cdot e^{-\frac{(x-\zeta)^{2}+(y-\eta)^{2}}{2 * \delta_{d}^{2}}} \\
& s(f(x, y), f(x-\zeta, y-\eta))=e^{\frac{(f(x, y)-f(x-\zeta, y-\eta))^{2}}{2 * \sigma_{r}^{2}}}
\end{aligned}
$$

Where $\delta_{d}=\sqrt{\ln 2 \cdot \lambda_{c} / 2}=\left(\sqrt{\ln 2 / \pi} \cdot \lambda_{c}\right)^{-1}$, and $\lambda_{c}$ is the cut-off length $\left(\lambda_{c}=2^{n+1} \cdot \Delta x\right.$ is sample interval and $\mathrm{n}$ is the decomposition layer-number). In this way the transmission characteristic of the filter is the same as the standard Gaussian filter; in this paper, $\delta_{r}=\mid$ med $\mid$.

\section{SimUlation}

We generate a $512 \times 512$ point random surface (shown in Fig.5.), with four added rectangular holes of the depth $0.1 \mu \mathrm{m}$, and the sampling interval $\Delta x=1$. A surface with additive noise $(\mathrm{SNR}=0.001 \mathrm{db})$ is shown in Fig.6. For DT-CWT, the decomposition level $n=5$, so the Gaussian filter and B-spline filter Cutoff wavelength $\lambda_{c}=2^{n+1}=64$.

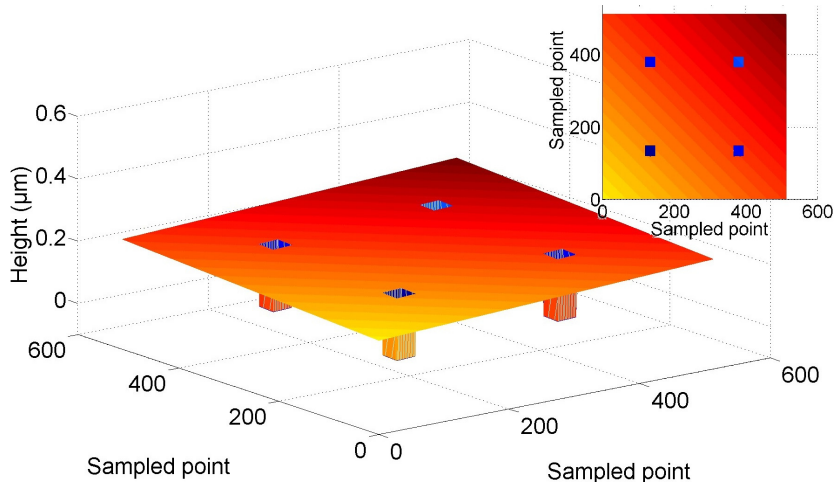

Fig.5. Simulated surface.

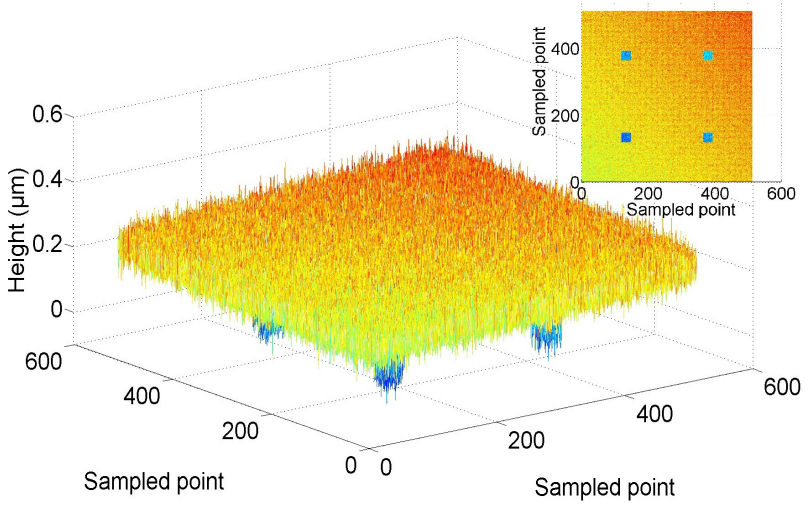

Fig.6. Additive noise surface. 
The Gaussian robust filter [11] and the B-spline filter are common methods of reference characterization in engineering, by which the reference surfaces are shown in Fig.7. and Fig.8. And reference surface, extracted by DT-CWT, is shown in Fig.9. Comparing to the original datum, reference surface extracted by the Gaussian robust filter has a greater boundary distortion and surface distortion which is pulled down at the position of the rectangular holes. When using the B-spline filter, the reference is also distorted at the position of the rectangular hole. And the reference surface through the DT-CWT is also affected by the hole, which is shown in Fig.9. These phenomena will highly influence the 3-D parameter's calculation. Then, Fig.10. demonstrates the merits and flexibility of the new method, which not only eliminates the boundary effect, but also suppresses outlier distortion. Its reference is flat and smooth, and is almost close to the simulation of the ideal reference.

To better explain the cause of the different reference, Fig. 11. presents the $\mathrm{X}$ direction profiles of each reference.

As seen from Fig.11. , the references extracted by the Gaussian robust filter and the B-spline filter are very close, and the only difference between them is that the boundary distortion exists in the reference through the Gaussian robust filter, but not the B-spline filter. When using DT-CWT, the distortion area is much larger than with other methods. Obviously, the reference surface by the improved filtering tends to approach the ideal surface.

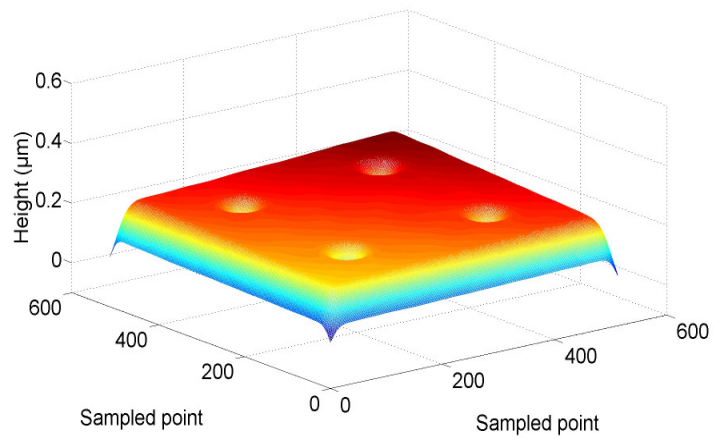

Fig.7. The reference surface extracted by Gaussian robust Filtering.

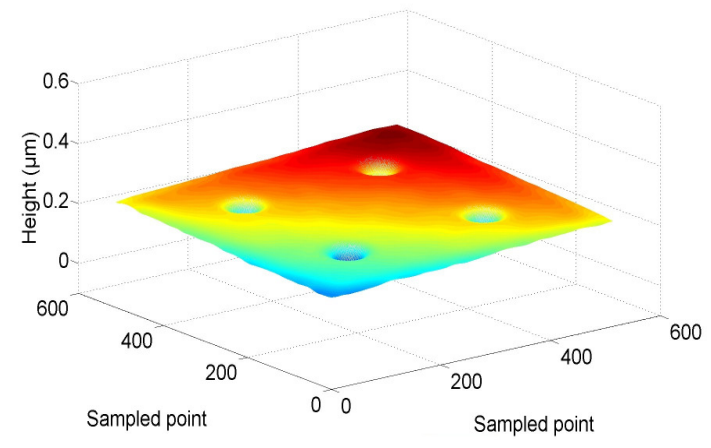

Fig.8. The reference surface extracted by B-spline filter.

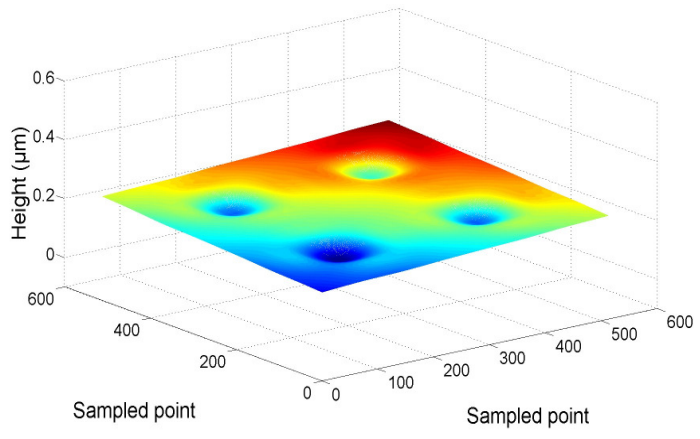

Fig.9. The reference surface extracted by DT-CWT.

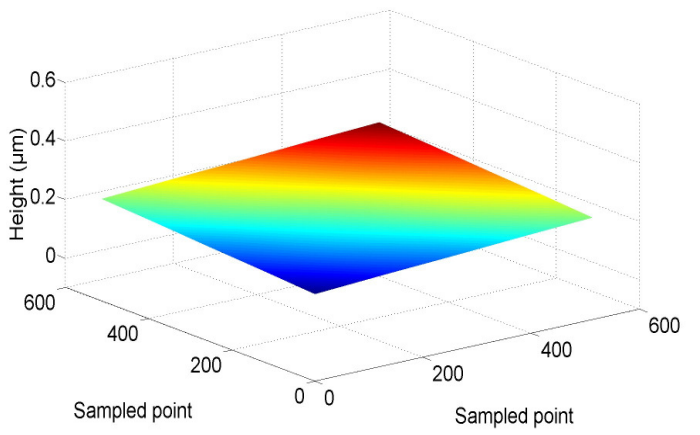

Fig.10. The reference surface extracted by the new algorithm.

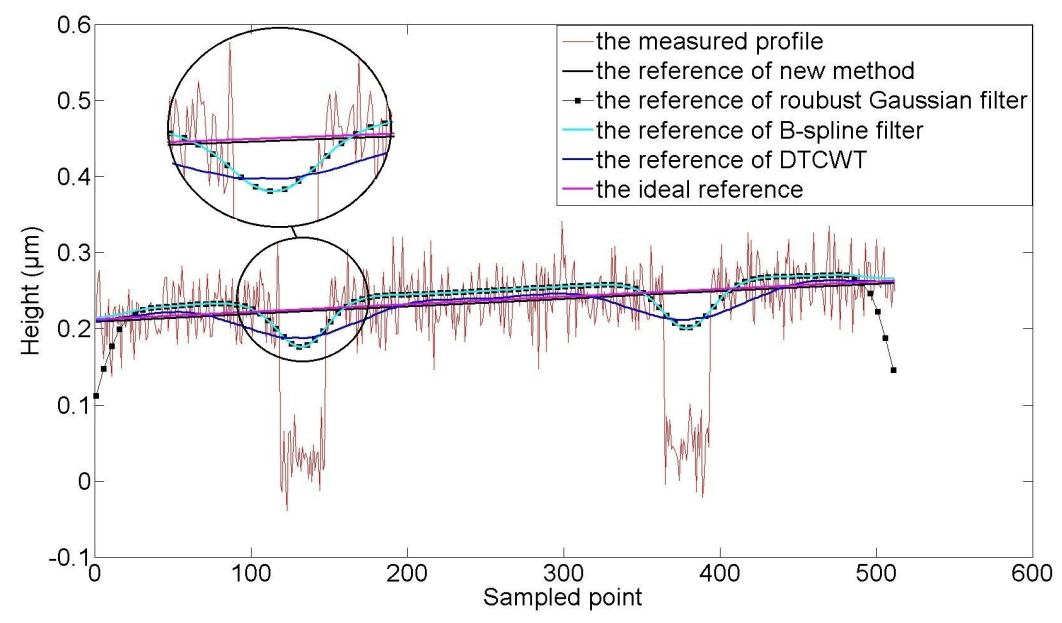

Fig.11. the $\mathrm{X}$ direction profiles of the each reference. 


\section{A. Example 1}

\section{CASE STUDY}

Fig.12. shows AFM data of a fine grinding surface with obvious abnormal features. This data was obtained by sampling $221 \times 217$ points with an interval of $0.5 \mu \mathrm{m}$. The optimal decomposition level (level 6) was used throughout the process. Therefore, $\delta_{d}=4.71$.

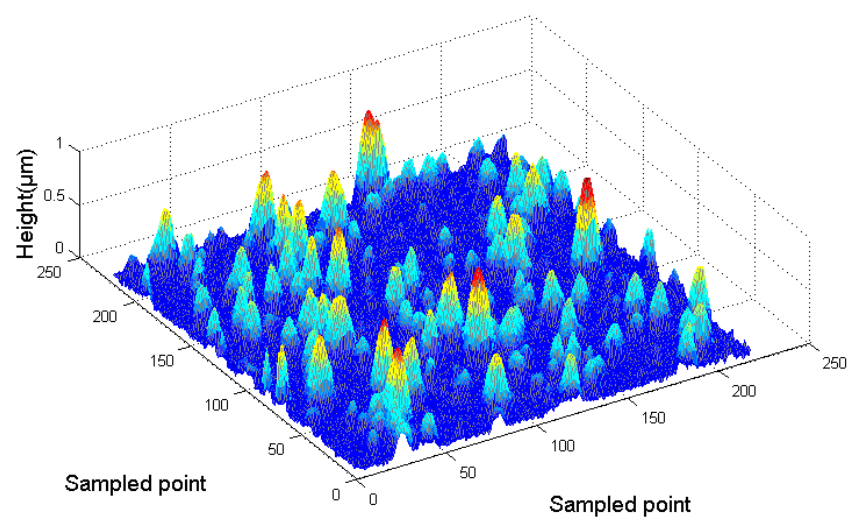

Fig.12. The measured surface.

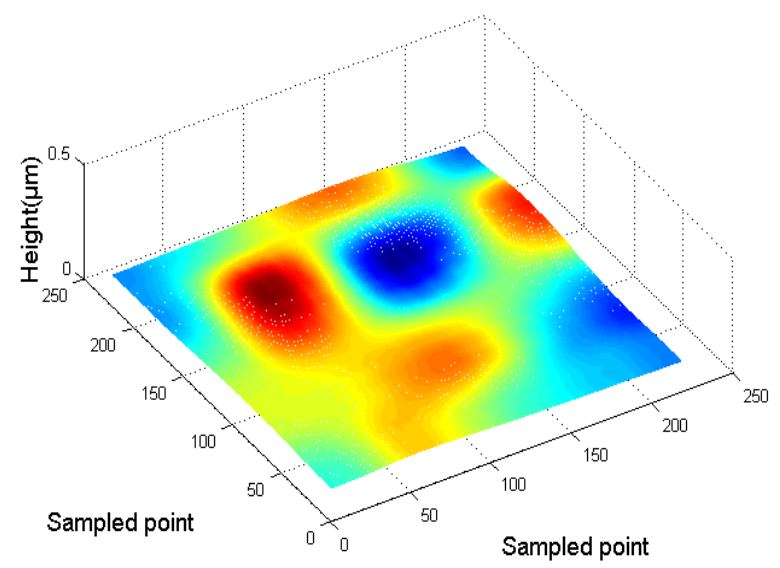

Fig.13. Reference of DT-CWT.

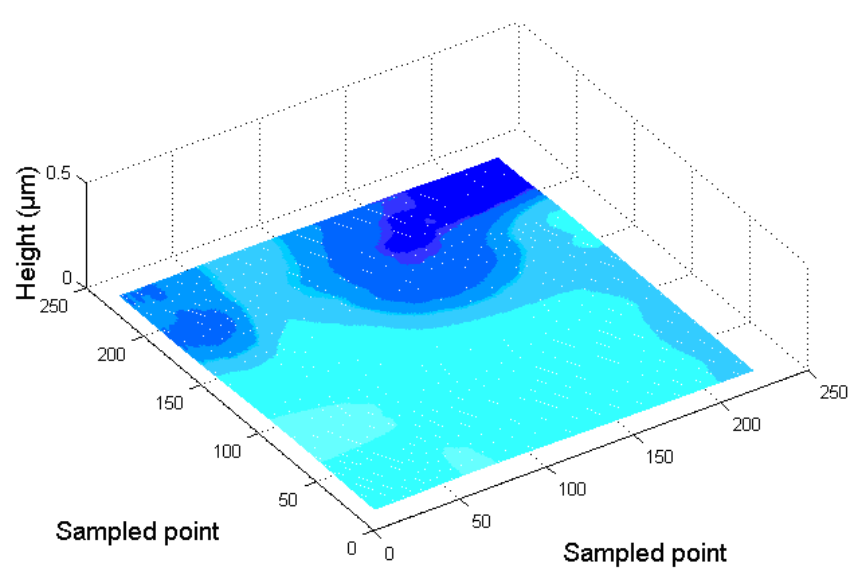

Fig.14. Reference of the new algorithm.

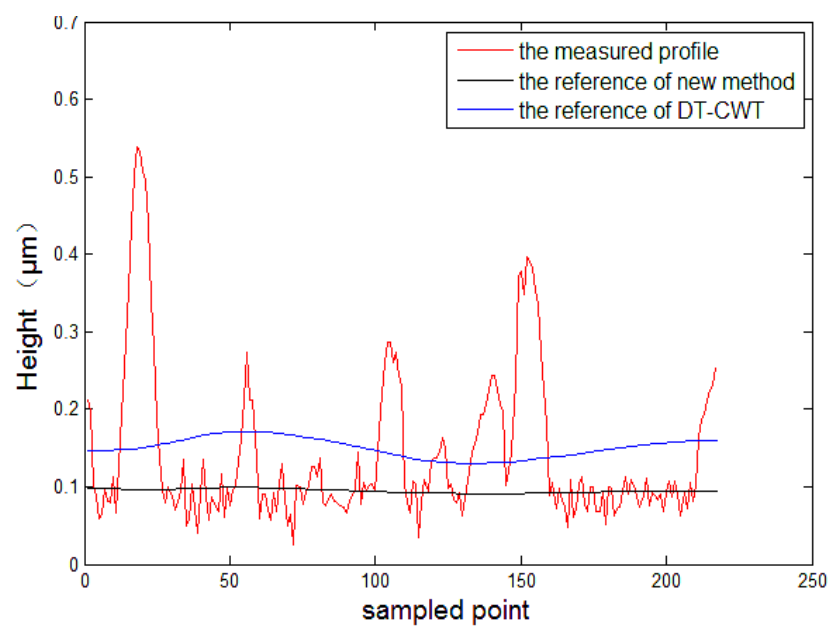

Fig.15. Comparison of reference height using different method (where $\mathrm{x}$ position is 20).

Fig.12. shows the measured surface, in which the abnormal features are in red and obvious. Fig.13. shows the references surface of DT-CWT, and it is affected at the region where the outliers exist. This is due to sensitiveness of DT-CWT to the outliers. In Fig.14., it can be seen that the reference surface of the improved method is smoother. However, the biggest difference between DT-CWT and the improved method is shown in Fig.15. The reference of DT-CWT is substantially higher than the reference of the improved algorithm, and the reference surface of the improved method does not move towards the peaks. In practice, the distortion and the improper location of conventional filtered surface would influence the final parametric assessment. By using the new method, the reference is improved, more reliable, and better at approximating reality.

\section{B. Example 2}

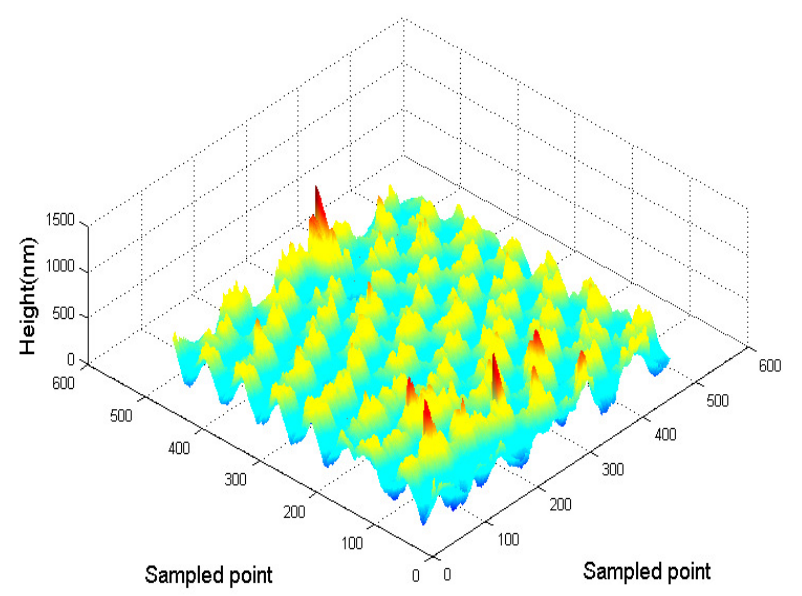

Fig.16. Measured surface with interval of $19 \mathrm{~nm}$ in which, $\mathrm{x}$ and $\mathrm{y}$ represent sample point and $\mathrm{z}$ is height.

It is well known that the manufacture of ultra-precision surfaces leaves multi-scale topography signatures in the surface. In this example, the reference and the other scale-frequency components which make up the waviness are 
both distorted by freak features. The new method also helps to characterize other scale-frequency components. Fig.16. shows a surface of porous anodic alumina containing obvious waviness components and the abnormal features shown in Fig.16. are in red.

Fig.17 represents the scale-frequency components of DT-CWT seriously distorted by the abnormal features and the influenced region of components is in red and shifts towards the outliers. The filtered surface of the new method is smoother and close to the ideal surface in Fig.18. A sectional diagram of Fig.17. and Fig.18. is presented in Fig.19. It is clear that the waviness profile of DT-CWT is pulled up or down and the center of the waviness profile is not around the reference line which is red. However, the waviness profile of the new method invariably vibrates around the reference line because, in the conventional method, the residual influence of freak features changes the height of waviness profile. Therefore, the center of waviness deviates from the reference. Thus, the profile parameter of the improved method would be more reasonable and better at approximating reality.

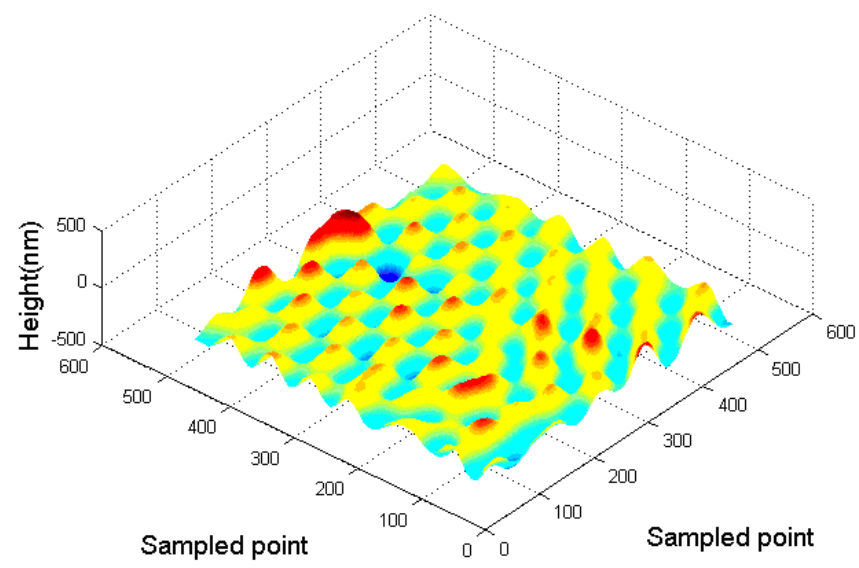

Fig.17. Waviness surface of DT-CWT.

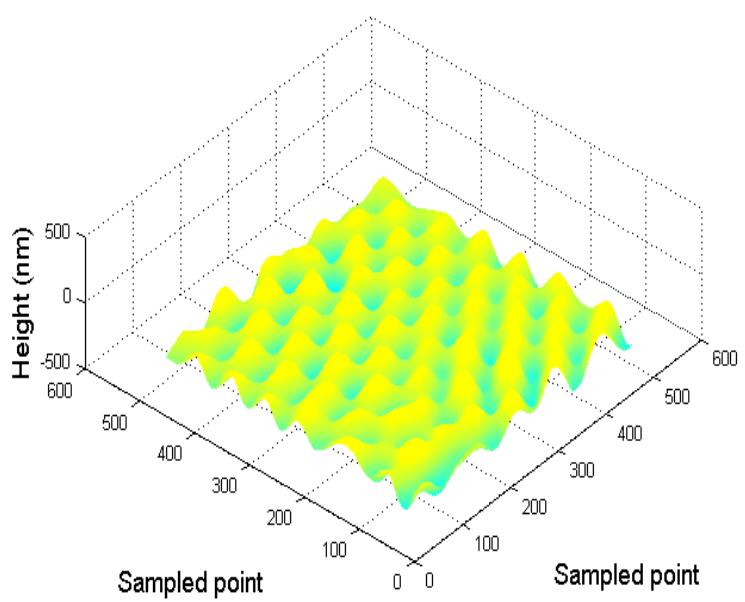

Fig.18. Waviness surface of the new algorithm.

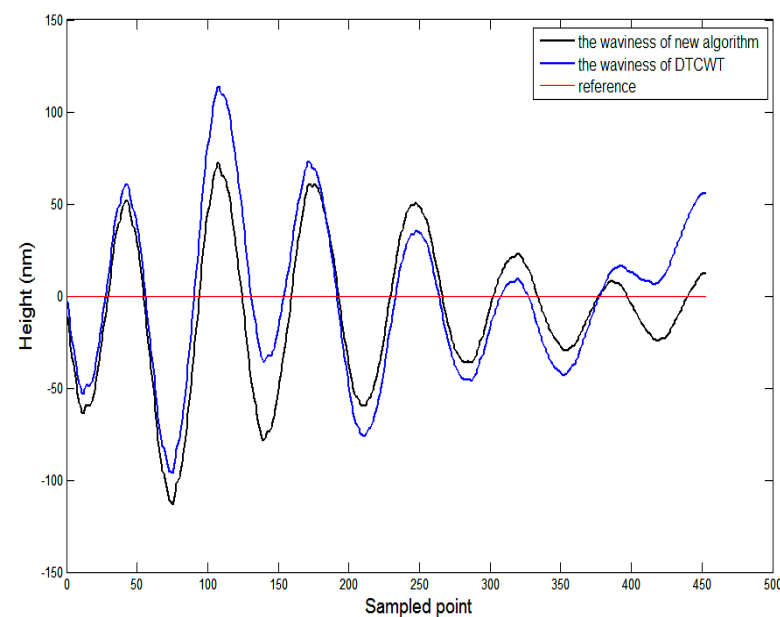

Fig.19. Comparison of waviness height using DT-CWT (the new method where $\mathrm{x}$ position is 200).

\section{CONCLUSION}

Due to the benefits of DT-CWT, such as shift invariance, directional selectivity, perfect reconstruction (PR), and limited redundancy, it has proven helpful and effective to separate the form, waviness and other components from an ultra-precision machined surface. However, application of DT-CWT was restricted by its sensitivity to freak features. This sensitivity was especially apparent at nano scales, where the morphology of surfaces is more complex and often contains pits, grooves and peaks. In this paper, we introduce an improved bilateral filtering to DT-CWT, which we used in image processing for edge-preservation. To verify the feasibility and accuracy of the proposed method, a PC simulation was used. Our results show that DT-CWT is superior to the conventional methods, but the new method provides the best reference and waviness, in every respect. In conclusion, as real ultra-precision machined surfaces are analyzed, tests of our new method indicate that the accuracy of the reference and waviness parameters exceeds the accuracy of conventional DT-CWT.

\section{ACKNOWLEDGEMENTS}

This work is supported by the National Natural Science Foundation of China (No. (51175085) and the National Youth Natural Science Foundation of China (No. 51205062 and No. 51205063). We are grateful to the center of Precision Technologies of School of Engineering at Fuzhou University for experimental assistance. Thanks to Dr. Shun C. Zhong for his instructive advice on my thesis.

\section{REFERENCES}

[1] Qinghu Chen, Zhu Li. (1998). The surface roughness wavelet assessed baseline. Acta Metrologica Sinica, 19 (4), 254-257.

[2] Wenhan Zeng. (2005). Tree complex wavelet surface analysis model and the process morphology identification method research. Dissertation, Huazhong University of Science and Technology, Wuhan, China.

[3] Josso, B., Burton, D.R., Lalor, M.J. (2002). Frequency 
normalized wavelet transform for surface roughness analysis and characterization. Wear, 252 (5-6), 491-500.

[4] Raja, J., Muralikrishnan, B., Shengyu Fu. (2002). Recent advances in separation of roughness, waviness and form. Precision Engineering, 26 (2), 222-235.

[5] Shengyu Fu, Muralikrishnan, B., Raja, J. (2003). Engineering surface analysis with different wavelet bases. Journal of Manufacturing Science and Engineering, 125 (4), 844-852.

[6] Jiang, X.Q., Blunt, L., Stout, K.J. (2001). Lifting wavelet for three-dimensional surface analysis. International Journal of Machine Tools and Manufacturing, 41 (13-14), 2163-2169.

[7] Kingsbury, N. (1999). Image processing with complex wavelets. Philosophical Transactions of the Royal Society A, 357, 2543-2560.

[8] Jiang, X.Q., Blunt, L. (2004). Third generation wavelet for the extraction of morphological features from micro and nano scalar surfaces. Wear, 257 (12), 1235-1240.

[9] Tomasi, C., Manduchi, R. (1998). Bilateral filtering for gray and color images. In International Conference on Computer Vision, 4-7 Jan. 1998. IEEE, 839-846.

[10] Selesnick, I.W. (2001). Hilbert transform pairs of wavelets bases. IEEE Signal Processing Letters, 8 (6), 170-173.

[11] International Organization for Standardization. (1996). Geometrical Product Specifications (GPS) - Surface texture: Profile method; Surfaces having stratified functional properties - Part 1: Filtering and general measurement conditions. ISO 13565-1:1996.

Received May 28, 2013. Accepted April 11, 2014. 